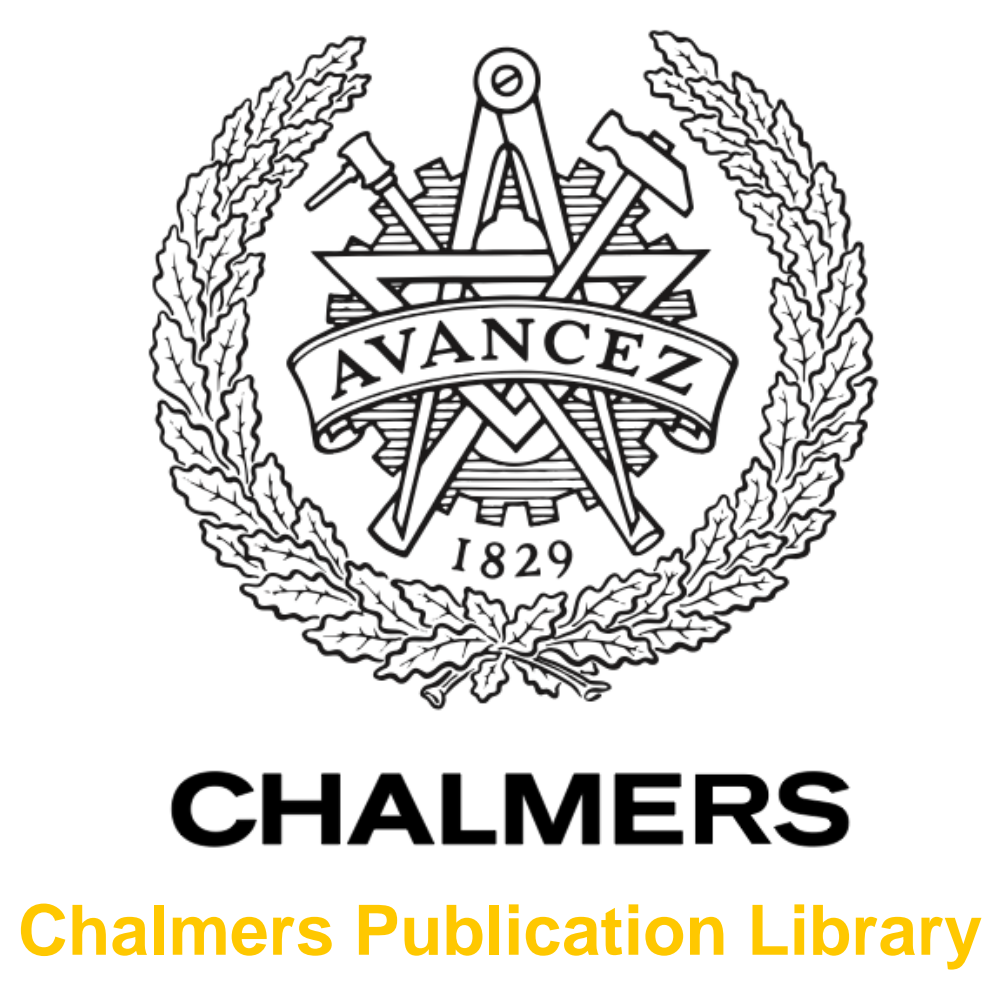

\author{
Probabilistic Threat Assessment and Driver Modeling in Collision Avoidance \\ Systems
}

This document has been downloaded from Chalmers Publication Library (CPL). It is the author's version of a work that was accepted for publication in:

IEEE Intelligent Vehicles Symposium, 5-9 June, 2011, Baden-Baden, Germany (ISSN: 19310587)

Citation for the published paper:

Sandblom, F. ; Brännström, M. (2011) "Probabilistic Threat Assessment and Driver

Modeling in Collision Avoidance Systems". IEEE Intelligent Vehicles Symposium, 5-9 June,

2011, Baden-Baden, Germany pp. 914-919.

http://dx.doi.org/10.1109/IVS.2011.5940554

Downloaded from: http://publications.lib.chalmers.se/publication/144748

Notice: Changes introduced as a result of publishing processes such as copy-editing and formatting may not be reflected in this document. For a definitive version of this work, please refer to the published source. Please note that access to the published version might require a subscription.

Chalmers Publication Library (CPL) offers the possibility of retrieving research publications produced at Chalmers University of Technology. It covers all types of publications: articles, dissertations, licentiate theses, masters theses, conference papers, reports etc. Since 2006 it is the official tool for Chalmers official publication statistics. To ensure that Chalmers research results are disseminated as widely as possible, an Open Access Policy has been adopted.

The CPL service is administrated and maintained by Chalmers Library. 


\title{
Probabilistic Threat Assessment and Driver Modeling in Collision Avoidance Systems
}

\author{
Fredrik Sandblom and Mattias Brännström
}

\begin{abstract}
This paper presents a probabilistic framework for decision-making in collision avoidance systems, targeting all types of collision scenarios with all types of single road users and objects. Decisions on when and how to assist the driver are made by taking a Bayesian approach to estimate how a collision can be avoided by an autonomous brake intervention, and the probability that the driver will consider the intervention as motivated. The driver model makes it possible to initiate earlier braking when it is estimated that the driver acceptance for interventions is high. The framework and the proposed driver model are evaluated in several scenarios, using authentic tracker data and a differential GPS. It is shown that the driver model can increase the benefit of collision avoidance systems particularly in traffic situations where the future trajectory of another road user is hard for the driver to predict, e.g. when a playing child enters the roadway.
\end{abstract}

Index Terms-automotive safety, collision avoidance, threat assessment, driver modeling, autonomous braking

\section{INTRODUCTION}

Road traffic accidents are one of the world's largest public health problems. In the EU alone, traffic accidents cause approximately 1.8 million injuries and 43.000 fatalities each year [1]. To reduce these numbers, vehicle manufactures are developing systems that can detect hazardous traffic situations and actively assist road users in avoiding or mitigating accidents. Systems that assist drivers in avoiding collisions are becoming increasingly more common and are even being introduced as standard equipment in some passenger cars [2].

Collision avoidance (CA) systems can generally be divided into three layers, as illustrated in Fig. 1. Measurements from in-vehicle sensors, such as accelerometers, gyros, cameras and radars, are processed in the first layer and then interpreted in the second layer that makes decisions on when and how to assist the driver. The third layer executes the decision, e.g. by automatically applying the brakes of the vehicle.

Fig. 1. Collision avoidance systems can be divided into three layers. This paper focuses on decision-making using a driver model.

The measurements are associated with uncertainties and, consequently, as are the vehicle and object state estimates

F. Sandblom and M. Brännström are with the Dept. of Signals and Systems, Chalmers University of Technology, SE-412 96 Gothenburg, Sweden

F. Sandblom is also with the Dept. of Electrical \& Electronics Engineering, Volvo 3P - Product Development, SE-405 08 Gothenburg, Sweden fredrik. sandblom. 2@volvo. com

M. Brännström is also with the Dept. of Safety Electronics and Functions, Volvo Car Corporation, SE-405 31 Gothenburg, Sweden mbranns3evolvocars.com

The authors would like to thank the Strategic Vehicle Research and Innovation (FFI) programme and the Intelligent Vehicle Safety Systems programme for sponsoring this work. that are obtained in the sensor fusion layer [3]. A threat assessment algorithm utilizes these estimates to make predictions of road user trajectories. Based on these predictions, an assessment is performed to judge how hazardous the traffic situation is and decisions are made. The objective is to assist the driver in avoiding collisions, without triggering warnings or interventions that the driver may consider as unnecessary. Both the state estimates and the predictions are associated with uncertainties, which will affect the decisions and thus need to be treated properly.

In this paper, we discuss how measurement and prediction uncertainties can be managed in a CA system by using a Bayesian approach to decision-making. In Section II, the need for driver modeling in CA systems is discussed and a novel driver model is formulated under a few basic assumptions regarding driver behaviour. Section III outlines the problem formulation. Section IV gives an introduction to Bayesian risk and hypothesis testing, and a probabilistic decision-making framework, including the driver model, is proposed. In Section $\mathrm{V}$ the driver model and decision strategy is further defined and the evaluation framework is presented. In Section VI, we evaluate the framework on a few different types of collision scenarios, using both simulated and authentic sensor measurements. Finally, conclusions are presented in Section VII.

\section{DRIVER MODELING}

In order to assist drivers in avoiding collisions, interventions or warnings must be triggered while a collision is avoidable. In practice, this means that accidents that are autonomously avoided by a CA system possibly also could have been avoided by the driver. Thus, it is only the driver of the vehicle that can decide if an intervention was motivated. This highlights the need of incorporating a driver model in the CA system, such that vehicle safety can be further improved without triggering interventions that the driver could consider as disturbing.

\section{A. Related literature}

Goodrich and Boer [4] propose that CA systems should account for not only the capabilities of the vehicle and the sensor system, but also the autonomy and preferences of the driver. Benefit and cost functions are introduced to make decisions based on a tradeoff between the potential benefit of an intervention and the cost for disturbing the driver with an unnecessary intervention. Although the concept of using cost functions is appealing at a first glance, this concept has some potential drawbacks that are pointed out, e.g., in [5]. 
For example, the cost of an unnecessary intervention may be difficult to define and relate to the benefit of avoiding a potential collision. Hence, the behaviour of the CA system may be hard to predict and tuning of the system could become problematic.

McCall and Trivedi [6] propose that the probability that the driver intends to apply the brakes shall be estimated and that interventions shall be inhibited if the driver intends to brake. The intent to brake is predicted by using a camera that monitors the driver's pedal usage and a camera that monitor the driver's face. Although a foot camera may be used to predict if the driver intend to apply the brakes, it is probably difficult to predict if the driver intends to steer. The driver may also be drowsy or cognitively distracted, in which case the driver intent could be difficult to predict, even if the driver has placed the foot on the brake pedal. Moreover, in traffic situations where the driver intends to brake to avoid a severe collision, it is reasonable to assume that the driver may consider a brake intervention as motivated and thus not disturbing.

Rather than estimating the drivers's intent or the cost for disturbing the driver, we propose that the CA system shall estimate if the driver will consider an intervention as motivated. In this way, driver autonomy can be maintained by only allowing the system to act when it is estimated that the driver has a high acceptability for warnings or interventions.

\section{B. The driver as a collision avoidance system}

The purpose of the driver model is to obtain a measure that indicates if the driver judges that the situation is critical. Some basic assumptions on driver behavior are discussed and a driver model based on these assumptions is formulated. This model is not intended for explaining other aspects of driver behavior, nor for predicting the driver's intentions.

If we assume that all drivers desire to avoid collisions, the drivers (consciously or subconsciously) need to

1) observe both stationary objects and other road users,

2) make predictions of road user trajectories, and

3) plan driving manoeuvres considering these predictions.

These three objectives motivate that the driver can be modeled as a collision avoidance system, consisting of

1) a sensor based tracking system, combined with

2) a prediction model for other road users, and

3) a path planning algorithm.

\section{Driver-preferred safety margins}

It is reasonable to assume that all drivers plan manoeuvres with some (minimum) safety margins to assure that collisions are avoided. We argue that these (physical) safety margins can be modeled by assuming that the driver is subconsciously aware that the driver's perception is limited [7] and that the driver's predictions of road user trajectories are somewhat uncertain, especially for some types of road users, such as

- wild animals,

- playing children, and

- vehicles that are skidding out of control.
Consequently, drivers are expected to plan manoeuvres with safety margins that increase with observation and prediction uncertainties. We argue that drivers also have safety margins in terms of vehicle dynamics and that these margins can be expressed as thresholds for "severe manoeuvres".

\section{Estimating driver acceptability for interventions}

We assume that drivers consider interventions as justified if they are triggered when the driver judges that the physical safety margins cannot be maintained; not even by using a severe evasive manoeuvre. That is, an intervention is motivated if triggered after the driver has passed a subjective "point-of-no-return" and judges that the traffic situation is critical. Under these assumptions, we conclude that:

\section{An intervention is justified if the driver (modeled as a CA system) judges that the traffic situation is critical when the intervention is triggered.}

In case the driver is distracted, we assume that once an intervention is triggered, the driver will shortly observe the threat and judge if the intervention was justified. The objective for the driver model subsystem (see Fig. 1) is to estimate the probability that the driver will consider an intervention as justified. Or equivalently, the probability that driver, modeled as a CA system with uncertain object state estimates and predictions, judges that the situation is critical.

\section{PROBLEM FORMULATION}

Let $\mathbf{x}$ be a state vector that represents the state (i.e. position, size, velocity etc.) of a vehicle hosting the CA system, and of a single obstacle, e.g. another vehicle or a pedestrian. Using the posterior state distribution $p(\mathbf{x} \mid \mathbf{y})$, given sensor data, $\mathbf{y}$, an intervention decision rule for avoiding accidents is desired. When making decisions in CA systems, there is always a trade-off between a high probability of a successful intervention that avoids or mitigates a collision, and the risk of annoying the driver. The latter risk can be assumed to vary and should thus be assessed in real-time, which raises two questions: Firstly, how can the risk be modeled and, secondly, what decision strategy is suitable when both the state estimate and trajectory predictions of road users are associated with uncertainties? Section IV aims to answer both questions by designing a driver model and a threat assessment algorithm that estimates if and how a collision can be avoided by autonomous braking, such that well proven decision rules can be employed and jointly evaluated, as shown in Fig. 1.

The driver is modeled as a CA system using measurements $\mathbf{z}$, i.e., what the driver can see. The goal of the driver is thus, in terms of a CA system, to calculate $p(\mathbf{x} \mid \mathbf{z})$ and make a control decision based on this distribution.

\section{PRoBABILISTIC DECISION-MAKING}

A robust decision rule must treat several sources of uncertainties. Firstly, the estimates of the state $\mathbf{x}$ originates from a tracking system with uncertainties. Secondly, the near future contains many possible outcomes, i.e., the prediction uncertainties are often of vital importance. Finally, 
the different accident scenarios and type of obstacles which a general collision avoidance system should handle are countless. Fig. 2 illustrates a scenario with many possible outcomes. Addressing these uncertainties motivate the need for a probabilistic approach to decision-making.

Fig. 2. Uncertainties in the tracking system estimate $\mathbf{x}_{t}$ at time $t$, and prediction uncertainties during the time interval $T$, affect the estimate of the predicted state $\mathbf{x}_{t+T}$. The vehicle hosting the CA system can be represented by any type of automotive vehicle, e.g. a car, a motorcycle, or a truck as illustrated in this scenario.

Several probabilistic decision-making algorithms have previously been proposed, e.g. [5], [8], [9], [10], [11]. The risk of a false alarm is typically balanced with the confidence of the necessity of a particular action. Rather than making this trade-off, we propose to use a driver model to describe when the drawbacks with an intervention arguably are negligible from the drivers perspective. The need for immediate braking can then be evaluated separately using the same robust approach as in [10]. Two indicator functions and their corresponding hypotheses forms the basis of the strategy:

$$
\begin{aligned}
& H_{0}: I_{H}(\mathbf{x})=0: \text { Driver does not accept intervention } \\
& H_{1}: I_{H}(\mathbf{x})=1: \text { Driver accepts intervention } \\
& \mathscr{H}_{0}: I_{\mathscr{H}}(\mathbf{x})=0: \text { Immediate braking is not needed } \\
& \mathscr{H}_{1}: I_{\mathscr{H}}(\mathbf{x})=1: \text { Immediate braking is needed }
\end{aligned}
$$

If the hypothesis probabilities can be calculated, the decision rule shown in Appendix I can be used to choose one hypothesis over another. Details can be found in most textbooks e.g. [12]. Two decision rules are needed, the driver acceptance decision and the decision for initiating autonomous braking. However, to calculate the former we will need to model also the driver decision, thereby introducing a third decision-rule.

\section{A. The driver acceptance decision}

For a given traffic situation, the indicator function

$$
I_{H}\left(\mathbf{x} \mid H_{i}\right)=i, i \in\{0,1\}
$$

determines if the driver accepts an intervention. The actual state $\mathbf{x}$ is unknown to the system but the distribution $p(\mathbf{x} \mid \mathbf{y})$ can be used to calculate the hypothesis probability

$$
\operatorname{Pr}\left\{H_{1} \mid \mathbf{y}\right\}=\int I_{H}(\mathbf{x}) p(\mathbf{x} \mid \mathbf{y}) d \mathbf{x}=\mathbb{E}\left[I_{H}(\mathbf{x}) \mid \mathbf{y}\right] .
$$

Thus, by marginalizing the tracking system uncertainties, decision rule (20) can be used to determine if an intervention is accepted or not. It remains however to define $I_{H}(\mathbf{x})$, the function which tell us if the driver will accept an intervention. We assume an intervention is acceptable if the driver would have acted (if aware of the situation) e.g., when deciding upon $\mathscr{H}_{1}$.

\section{B. Intervention decision for driver-initiated braking}

The driver is modeled as a sensor aware of its shortcomings in the context of driving, and the drivers sense of safety is modeled as choice between hypotheses $\mathscr{H}_{0}$ and $\mathscr{H}_{1}$. Let $\mathscr{O}$, e.g., represent an object, e.g., another vehicle or a pedestrian and $\tilde{\mathbf{w}}$ be the driver's error when predicting its motion. The driver model marginalizes these errors in order to calculate the hypothesis probability,

$$
\begin{aligned}
\operatorname{Pr}\left\{\mathscr{H}_{1} \mid \mathbf{z}, \mathscr{O}\right\} & =\iint I_{\mathscr{H}}(\mathbf{x}, \tilde{\mathbf{w}}, \mathscr{O}) p(\mathbf{x}, \tilde{\mathbf{w}} \mid \mathbf{z}, \mathscr{O}) d \mathbf{x} d \tilde{\mathbf{w}} \\
& =\mathbb{E}\left[I_{\mathscr{H}}(\mathbf{x}) \mid \mathbf{z}, \mathscr{O}\right]
\end{aligned}
$$

where $p(\mathbf{x}, \tilde{\mathbf{w}} \mid \mathbf{z}, \mathscr{O})$ describes the driver uncertainties when observing and predicting the future trajectory of the object $\mathscr{O}$. The driver's sense of safety decision can thus be modeled:

$$
\frac{\operatorname{Pr}\left\{\mathscr{H}_{1} \mid \mathbf{z}, \mathscr{O}\right\}}{\operatorname{Pr}\left\{\mathscr{H}_{0} \mid \mathbf{z}, \mathscr{O}\right\}} \underset{I_{H}(\mathbf{x})=0}{\stackrel{I_{H}(\mathbf{x})=1}{\gtrless}} c_{1}(\mathscr{O}),
$$

where $c_{1}(\mathscr{O})$ is a threshold for selecting $\mathscr{H}_{1}$ over $\mathscr{H}_{0}$, i.e., the driver decides that the situation is critical.

In summary: According to the argumentation in Section II-D, an intervention is accepted if the driver judges that a situation is critical. This decision, $I_{H}(\mathbf{x})$, is modeled by equation (6). However, as $\mathbf{x}$ is not fully known by the sensor fusion system, nor is $I_{H}(\mathbf{x})$. Instead, the probability that the driver accepts an intervention is calculated [see (4)]. Consequently, it can be determined if an intervention should be allowed by testing

$$
\frac{\operatorname{Pr}\left\{H_{1} \mid \mathbf{y}\right\}}{\operatorname{Pr}\left\{H_{0} \mid \mathbf{y}\right\}} \underset{H_{0}}{\stackrel{H_{1}}{\gtrless}} c_{2}(\mathscr{O}),
$$

where $c_{2}(\mathscr{O})$ is a threshold for selecting $H_{1}$ over $H_{0}$.

\section{Intervention decision for autonomous braking}

In the previous section it was shown how the driver decides for an intervention. Naturally, since the driver is modeled as a CA system, the process of determining whether a CA system should intervene is analogous. For notational convenience we assume that the indicator function $I_{\mathscr{H}}(\mathbf{x})$ could be applied to both the the driver model and the autonomous system.

Assume that there is a threat assessment function $g(\cdot)$ that estimates if immediate braking is needed in order to avoid a collision, such that

$$
I_{\mathscr{H}}(\mathbf{x})= \begin{cases}1, & g(\mathbf{x}) \geq \text { some threshold } \\ 0, & \text { else. }\end{cases}
$$

The probabilities for the corresponding hypotheses [see (2)] are now readily calculated the same way as in the driver model [see (5)]:

$$
\operatorname{Pr}\left\{\mathscr{H}_{1} \mid \mathbf{y}\right\}=\mathbb{E}\left[I_{\mathscr{H}}(\mathbf{x}) \mid \mathbf{y}, \mathscr{O}\right]
$$

and the intervention decision rule becomes

$$
\frac{\operatorname{Pr}\left\{\mathscr{H}_{1} \mid \mathbf{y}\right\}}{\operatorname{Pr}\left\{\mathscr{H}_{0} \mid \mathbf{y}\right\}} \underset{\mathscr{H}_{0}}{\stackrel{\mathscr{H}_{1}}{\gtrless}} c_{3}(\mathscr{O}),
$$

where $c_{3}(\mathscr{O})$ is a threshold for selecting $\mathscr{H}_{1}$ over $\mathscr{H}_{0}$. 


\section{DESIGN ChOICES AND IMPLEMENTATION}

In the previous section, two decision rules were designed to answer the following questions:

1) Does the driver accept an intervention?

2) Is there a need for an intervention?

Only if the answer to both these questions are 'yes' should the system take action. Many threat assessment algorithms known from literature could be used in this setting and benefit from a reduced false alarm rate, but the driver model allows for an advantageous design change: under hypothesis $H_{1}$, an intervention may be initiated at an earlier stage to increase the benefit of the intervention without disturbing the driver. This can be readily achieved by significantly lowering the loss [see (18)] for making a decision to intervene [see (10)] when the driver is believed to accept an intervention. As shown in Appendix II, this can significantly increase the capability to completely avoid an accident.

\section{A. Driver model}

In Section II, it is argued that the driver can be modeled as a CA system. Hence, we can use an algorithm previously proposed in [13] for assessing how the driver can either steer, brake or accelerate to avoid a collision with an arbitrary obstacle. The lateral or longitudinal acceleration required to avoid an accident by executing a manoeuvre $M$ is

$$
a_{\text {driver }}^{\text {req }}=g(\mathbf{x}, \tilde{\mathbf{w}}, \mathscr{O}, M),
$$

where $g(\cdot)$ is the algorithm from [13]. Recall from Section IV-A that the indicator function $I_{\mathscr{H}}$ defines when a situation is critical to the driver, i.e., the driver will act. Our conservative model states that a situation is critical if the required acceleration needed to avoid crash $a_{\text {driver }}^{\text {req }}$ with respect to manoeuvre $M$, is greater than a threshold $a_{\text {driver }}^{\max }(M)$, i.e.,

$$
I_{\mathscr{H}}^{\text {Driver }}(\mathbf{x}, \tilde{\mathbf{w}}, \mathscr{O})=\left\{\begin{array}{ll}
1, & g(\mathbf{x}, \tilde{\mathbf{w}}, \mathscr{O}, M) \geq a_{\text {driver }}^{\max }(M), \forall M . \\
0, & \text { else }
\end{array} .\right.
$$

The situation as a whole is critical if the situation is critical with respect to all manoeuvres $M$. A sufficiently high threshold $a_{\text {driver }}^{\max }$ corresponds to a conservative driver model. That is, most drivers would agree that a situation is indeed critical when the model says so. The thresholds used to evaluate the decision-making strategy are listed in Section V-D. The relation between driver measurements and the state is assumed linear with additive Gaussian noise:

$$
\mathbf{z}=\mathbf{x}+\mathbf{v}, \quad \mathbf{v} \sim \mathscr{N}\left(0, P_{\mathbf{v}}\right) .
$$

\section{B. Threat assessment}

Given that the driver accepts an intervention, autonomous braking will be triggered by the system when the required deceleration $a_{\text {system }}^{\text {req }}$ to avoid a collision crosses a threshold, preferably below the maximum capacity of the brake system to leave some margin for avoidance. The indicator function corresponding to the intervention hypotheses $\mathscr{H}_{0}, \mathscr{H}_{1}$ is

$$
I_{\mathscr{H}}^{\text {System }}(\mathbf{x}, \mathbf{w}, \mathscr{O})= \begin{cases}1, & g\left(\mathbf{x}, \mathbf{w}, \mathscr{O},{ }^{\prime} \text { Brake' }\right) \geq a_{\text {system }}^{\text {max }}(\mathscr{O}) \\ 0, & \text { else }\end{cases}
$$

where $\mathbf{w}$ is the CA system's prediction noise with respect to object $\mathscr{O}$. The hypothesis probabilities [see (9)] can now be calculated and the intervention decision rule [see (10)] can be applied.

\section{Implementation}

The hypothesis tests preceding an intervention can be performed after calculating the integrals (4), (5) and (9). Finding analytical solutions to these integrals is rarely possible, which motivates the usage of approximative methods. A straightforward approach is to generate samples $\mathbf{x}^{(i)}$ from $p(\mathbf{x})$ and use Monte Carlo integration to approximate $\operatorname{Pr}\{H\}$ :

$$
\operatorname{Pr}\{H\}=\int I_{H}(\mathbf{x}) p(\mathbf{x}) d \mathbf{x} \approx \frac{1}{N} \sum_{i=1}^{N} I_{H}\left(\mathbf{x}^{(i)}\right)=\operatorname{Pr}\{H\}_{N},
$$

where $\operatorname{Pr}\{H\}_{N} \stackrel{a . s}{\longrightarrow} \operatorname{Pr}\{H\}$ as $N \rightarrow \infty$, see e.g. [15]. These methods yield high performance but are computationally costly; as a reference for evaluating more cost-efficient methods, we use $N=1000$ for a state vector of length 8 , including prediction uncertainties.

Less demanding numerical integration methods such as the family of sigma point techniques evaluated in [16], or the recent cubature integration rule [17], are unfortunately not well suited for integrating over binary functions. However, our studies show that they perform well in approximating the first two moments of $p\left(a^{r e q} \mid \mathbf{y}\right)$, for our choice of $g(\cdot)$. Hypothesis probabilities are thus readily calculated analytically under a Gaussian assumption on this distribution.

A third approach, which is the method used in this paper, is to use a grid of deterministically chosen sample points with associated weights to approximate the integral as a weighted sum of evaluations,

$$
\operatorname{Pr}\{H\} \approx \sum_{i=1}^{L} w_{i} I_{H}\left(\mathbf{x}^{(i)}\right) .
$$

Grid methods are only exact for discrete state spaces. However, in Section VI a grid of size $L=49$, using weights $w_{i} \propto p\left(\mathbf{x}^{(i)} \mid \mathbf{y}\right)$, perform very well. The evaluation points are selected to be the union of the sigma points generated from an Unscented transform with $\kappa=0,1-n_{x}, 1-\frac{n_{x}}{3}$ respectively. $n_{x}$ is the length of the state vector and the interpretation of $\kappa$ is explained e.g. in [18].

We suggest using an evasive braking manoeuvre from the high-valued tail of $p\left(a_{\text {system }}^{\text {req }} \mid \mathbf{y}\right)$. This will initially yield distinct braking, but soon the distribution will reflect the effects of the action, and the predictive control strategy may be updated to decrease the brake force towards the end of the manoeuvre.

\section{Parameters}

The acceleration required to avoid a collision using four different types of manoeuvres, \{Brake, Accelerate, Turn left, Turn right $\}$ is calculated. Drivers are assumed to consider accelerations greater than $a_{\text {driver }}^{\max }$ as severe manoeuvres, as shown by the selected thresholds in Table I. The thresholds have been selected $a d$ hoc and are used in 
Section VI to illustrate some interesting properties of the proposed decision-making framework. It is suggested that extensive testing both in field operational tests and on test tracks should be conducted to find a more suitable selection of thresholds, but such studies are out of scope for this paper. To further limit the scope of our analysis, we assume drivers only have difficulties in estimating the acceleration of other road users, whereas position, size and velocity estimates are highly accurate. Thus, all elements of $P_{\tilde{v}}$ are set to zero except for the acceleration variance, which is set to $4\left[\mathrm{~m}^{2} / \mathrm{s}^{4}\right]$.

TABLE I

THRESHOLDS USED IN SECTION VI

\begin{tabular}{llll}
\hline \hline$p(\mathbf{x} \mid \mathbf{y})=$ & $\mathscr{N}\left(\overline{\mathbf{x}}, P_{x}\right)$ & $\mathbf{z}$ & $\sim \mathscr{N}\left(\overline{\mathbf{x}}, P_{v}\right)$ \\
$a_{\text {system }}^{\text {max }}(\mathscr{O})$ & $8\left[\mathrm{~m} / \mathrm{s}^{2}\right]$ & $a_{\text {driver }}^{\max }(\mathscr{O}, M)$ & $7\left[\mathrm{~m} / \mathrm{s}^{2}\right] \forall M$ \\
$\mathscr{H}_{1}^{\text {system }}:$ & $a_{\text {system }}^{\text {req }} \geq a_{\text {system }}^{\text {max }}$ & $\mathscr{H}_{1}^{\text {driver }}:$ & $a_{\text {driver }}^{\text {req }} \geq a_{\text {driver }}^{\text {max }}$ \\
$H_{1}:$ & ${\operatorname{Pr}\left\{\mathscr{H}_{1}^{\text {driver }}\right\}}^{\operatorname{Pr}\left\{\mathscr{H}_{0}^{\text {driver }}\right\}}>c_{1}$ & $c_{1}(\mathscr{O})$ & 0.25 \\
\hline
\end{tabular}

In collision scenarios conducted with real vehicles, the state estimates $\overline{\mathbf{x}}, P_{\hat{x}}$ are provided by a tracker [14], using a further developed version of the radar sensor model presented in [3]. For simulated scenarios the estimates are set to the true position and an average covariance matrix. To predict vehicle trajectories we use a standard bicycle model [19] with jerk uncertainties affecting the curvature, $c_{0}$, and longitudinal acceleration, $a$, expressed in terms of vehicle limitations,

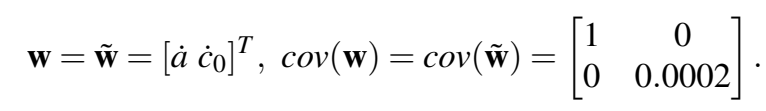

\section{RESULTS}

The driver model and the proposed decision-making framework is applied to the collision scenarios illustrated in Fig. 3, using the grid based implementation described in Section V-C.

Fig. 3. Collision scenarios with a lead vehicle, a playing child, a thrash can and a turning vehicle. The arrows in the rear end collision scenario illustrates scenarios with and without an evasive steering manoeuvre.

Results for an authentic rear-end collision scenario is presented in Fig. 4. For safety reasons, the lead vehicle is represented by a soft inflatable car $(3 \times 1.7 \mathrm{~m})$ which is attached to a trolley driven by a wire system at $50 \mathrm{~km} / \mathrm{h}$. The state of the soft car is estimated by using a differential GPS system. Measurement noise is then added to obtain measurements representative of a radar-based sensor system. In a first test, the driver of the host vehicle approaches the lead vehicle at $80 \mathrm{~km} / \mathrm{h}$ and performs a late evasive steering manoeuvre. Immediately prior to this manoeuvre it can be seen that the probability that the driver considers the traffic situation as critical increases significantly, but it decreases as soon as the driver initiates an evasive steering manoeuvre. In a second test, the host vehicle drives straight into the lead vehicle at $80 \mathrm{~km} / \mathrm{h}$ without braking or steering. The results in Fig. 4 show that the CA system can prevent an accident without disturbing the driver with unnecessary braking.
Fig. 4. Rear-end collision scenario with (top) and without (bottom) an evasive steering manoeuvre. The graphs show the probability that the driver will accept a brake intervention, $\operatorname{Pr}\left\{H_{1} \mid \mathbf{y}\right\}$ (solid), and the probability that braking is needed, $\operatorname{Pr}\left\{\mathscr{H}_{1} \mid \mathbf{y}\right\}$ (dashed). The dotted line shows the lateral acceleration (in [g]) of the host vehicle. The star marks the time ( $t=2 \mathrm{~s})$ when the vehicles collide if no evasive action is taken. At $t \approx 1.75 \mathrm{~s}$ (top), $\operatorname{Pr}\left\{\mathscr{H}_{1} \mid \mathbf{y}\right\}$ rises because the host vehicle passes close to the lead vehicle.

Fig. 5 depicts simulated scenarios where the driver is approaching either a playing child, or a thrash can, at $50 \mathrm{~km} / \mathrm{h}$. The driver is assumed to have similar estimates of the position of both objects, and knows that the trash can is stationary. However, since it is difficult for the driver to predict the child's future trajectory, the system detects that the driver has a higher acceptance for earlier autonomous braking than when approaching the thrash can.

Fig. 5. The probability that the driver will accept a brake intervention, $\operatorname{Pr}\left\{H_{1} \mid \mathbf{y}\right\}$ (solid), when approaching a thrash can (top) or a playing child (bottom) at $50 \mathrm{~km} / \mathrm{h}$. The star marks the time $(t=2 \mathrm{~s})$ when a collision occurs if no evasive action is taken. The dashed line shows the probability that immediate braking is needed, $\operatorname{Pr}\left\{\mathscr{H}_{1} \mid \mathbf{y}\right\}$. When approaching the child, $\operatorname{Pr}\left\{H_{1} \mid \mathbf{y}\right\}$ rises earlier than $\operatorname{Pr}\left\{\mathscr{H}_{1} \mid \mathbf{y}\right\}$ and thus, the CA system can use autonomous braking to prevent a collision without disturbing the driver.

In Fig. 6, an evaluation is performed in an intersection collision scenario using a radar-based sensor fusion system. In order to obtain realistic measurements, a real target vehicle was used. The state of the host vehicle was then simulated to drive at a higher speed $(50 \mathrm{~km} / \mathrm{h})$ than the actual speed, such that a collision situation was created without putting the drivers at risk. The results indicate that it is realistic that CA systems can be designed to autonomously avoid, or at least mitigate, this common type of collision without disturbing the driver with unnecessary braking.

Fig. 6. The probability that the driver accepts an intervention, $\operatorname{Pr}\left\{H_{1} \mid \mathbf{y}\right\}$ (solid), and the probability that braking is needed, $\operatorname{Pr}\left\{\mathscr{H}_{1} \mid \mathbf{y}\right\}$ (dashed), using data collected by a radar based sensor fusion system. The host vehicle (bottom) is approaching a turning vehicle (top). The star marks the time $(t=2 \mathrm{~s})$ when a collision occurs if no evasive action is taken.

\section{CONCLUSIONS}

In this paper, we presented a framework for decisionmaking in human centric collision avoidance (CA) systems. We introduced a driver model, resembling a CA system with uncertain input data, and showed that the use of this model has several appealing properties. Specifically, the driver model enables the safety system to perform earlier interventions in situations where the future trajectories of other road users are difficult for the driver to predict. Moreover, the proposed framework formally handles both measurement and prediction uncertainties, as well as the driver's subjective safety margins.

\section{APPENDIX I \\ BAYESIAN DECISION-MAKING}

Let there be two hypotheses; $\mathfrak{h}_{0}$ and $\mathfrak{h}_{1}$. Given some observations $\mathbf{y}$, we wish to make a decision regarding which hypothesis is true. Let $\alpha_{i}$ be the decision that hypothesis $\mathfrak{h}_{i}$ is the correct one and suppose we can express a loss 
$\lambda_{i j}=L\left(\alpha_{i} \mid \mathfrak{h}_{j}\right)$ for making the decision $\alpha_{i}$ given that the true state is $\mathfrak{h}_{j}$. The risk $R\left(\alpha_{i} \mid \mathbf{y}\right)$ is defined as the expected loss associated with a particular decision,

$$
R\left(\alpha_{i} \mid \mathbf{y}\right)=\sum_{j=0,1} \lambda_{i j} \operatorname{Pr}\left\{\mathfrak{h}_{j} \mid \mathbf{y}\right\}
$$

The minimum risk is achieved by choosing $\mathfrak{h}_{1}$ over $\mathfrak{h}_{0}$ if $R\left(\alpha_{1} \mid \mathbf{y}\right)<R\left(\alpha_{0} \mid \mathbf{y}\right)$, i.e. if

$$
\frac{p\left(\mathbf{y} \mid \mathfrak{h}_{1}\right)}{p\left(\mathbf{y} \mid \mathfrak{h}_{0}\right)}>\frac{\left(\lambda_{10}-\lambda_{00}\right)}{\left(\lambda_{01}-\lambda_{11}\right)} \frac{\operatorname{Pr}\left\{\mathfrak{h}_{0}\right\}}{\operatorname{Pr}\left\{\mathfrak{h}_{1}\right\}} .
$$

This is equivalent to evaluating the well-known likelihood ratio. It may be convenient to evaluate the posterior probabilities rather than the likelihood, e.g in a particle filter implementation. Applying Bayes formula to the left hand side of equation (19) gives the equivalent rule

$$
\frac{\operatorname{Pr}\left\{\mathfrak{h}_{1} \mid \mathbf{y}\right\}}{\operatorname{Pr}\left\{\mathfrak{h}_{0} \mid \mathbf{y}\right\}} \underset{\mathfrak{h}_{0}}{\gtrless} \frac{\mathfrak{h}_{1}}{\lambda_{01}-\lambda_{11}}=c .
$$

The above relation suggests to choose $\mathfrak{h}_{1}$ over $\mathfrak{h}_{0}$ if the ratio $\operatorname{Pr}\left\{\mathfrak{h}_{1} \mid \mathbf{y}\right\} / \operatorname{Pr}\left\{\mathfrak{h}_{0} \mid \mathbf{y}\right\}$ exceeds a threshold $c$.

\section{APPENDIX II}

\section{Mitigation to AVOIDANCE}

Assume that a vehicle is driving at an initial speed, $v_{0}$, and that emergency braking is initiated such that the speed is reduced to $v_{c}$ before colliding with a stationary object. How much earlier, $\Delta t$, should the braking be initiated to avoid the collision? The deceleration will eventually reach a constant value, $a$, and the collision is avoided if $s=\frac{v_{c}^{2}}{2 a}$ extra meters are available. The time to travel this distance, before braking is initiated, is

$$
\Delta t=\frac{s}{v_{0}}=\frac{v_{c}^{2}}{2 a v_{0}} .
$$

Consequently, by applying the brakes $\Delta t$ seconds earlier, the required extra distance is gained and the collision is avoided.

For $v_{0}=15[\mathrm{~m} / \mathrm{s}]$ and $v_{c}=5[\mathrm{~m} / \mathrm{s}]$ (i.e., 54 and $18 \mathrm{~km} / \mathrm{h}$ ) on dry asphalt $\left(a=10\left[\mathrm{~m} / \mathrm{s}^{2}\right]\right)$, it is enough to brake $83[\mathrm{~ms}]$ earlier in order to fully avoid an accident. See Fig. 7 for more examples. Apparently, the required difference in timing to attain avoidance rather than mitigation can be very small.
Fig. 7 . The three curves show $\Delta t$ for three crash velocities, $v_{c}=20,15$, and $10[\mathrm{~km} / \mathrm{h}]$, top to bottom. Braking $\Delta t$ seconds earlier fully avoids the collision with the smallest possible margin. The result is valid for braking on dry asphalt and is invariant to the brake system ramp-up time.

\section{REFERENCES}

[1] "Annual statistical report," European Road Safety Observatory, 2008.

[2] M. Distner, M. Bengtsson, T. Broberg and L. Jakobsson, "City Safety - A system addessing rear-end collisions at low speeds," Proc. $21^{\text {th }}$ ESV Conf., paper 09-0371, 2009.

[3] J. Gunnarsson, L. Svensson, L. Danielsson and F. Bengtsson, "Tracking vehicles using radar detections," Proc. IEEE Intell. Veh. Symp., pp. 296302, 2007.

[4] M. Goodrich and E. Boer, "Designing human-centered automation: Tradeoffs in collision avoidance system design," IEEE Trans. Intell. Transp. Syst., vol. 1, no. 1, pp. 40-54, Mar. 2000.

[5] J. Hillenbrand, A.M. Spieker and K. Kroschel, "A multilevel collision mitigation approach - Its situation assessment, decision making and performance tradeoffs," IEEE Trans. Intell. Transp. Syst., vol. 7, no. 4, pp. 528-540, 2006.
[6] J. McCall and M. Trivedi, "Driver Behavior and Situation Aware Brake Assistance for Intelligent Vehicles," Proc. of the IEEE, vol. 95, no. 2, pp. 374-386, Feb. 2007

[7] D. Delorme and B. Song, "Human Driver Model for SmartAHS", Tech. Rep. UCB-ITS-PRR-2001-12, California PATH Program, Institute of Transportation Studies, University of California, Berkeley, April 2001.

[8] A. Eidehall and L. Petersson, "Statistical threat assessment for general road scenes using Monte Carlo sampling," IEEE Trans. Intell. Transp. Syst., vol. 9, no. 1, pp. 137-147, 2008.

[9] N. Kaempchen, B. Schiele and K. Dietmayer, "Situation assessment of an autonomous emergency brake for arbitrary vehicle-to-vehicle collision scenarios," IEEE Trans. Intell. Transp. Syst., vol. 10, no. 4, pp. 678-687, 2009.

[10] J. Jansson and F. Gustafsson, "A framework and automotive application of collision avoidance decision making," Automatica, Vol. 44, No. 9, pp. 2347-2351, 2008.

[11] M. Althoff, O. Stursberg and M. Buss, "Model-based probabilistic collision detection in autonomous driving," IEEE Trans. Intell. Transp. Syst., vol. 10, no. 2, pp. 299-310, 2009.

[12] R. O. Duda, P. E. Hart, and D. G. Stork, Pattern Classification, 2nd ed. New York: Wiley, 2001.

[13] M. Brännström, E. Coelingh and J. Sjöberg, "Model-based threat assessment for avoiding arbitrary vehicle collisions," IEEE Trans. Intell. Transp. Syst., , vol. 11, no. 3, pp. 658-669, Sept. 2010.

[14] F. Bengtsson, L. Danielsson, "Designing a real time sensor data fusion system with application to automotive safety", Tech. report no. R007/2008, Department of Signals and systems, Chalmers University of Technology, Gothenburg, April 2008.

[15] J. Geweke, (1996). Monte Carlo Simulation and Numerical Integration. In Handbook of Computational Economics (Vol. 1, pp. 731-800).

[16] Y. Wu et al. (2006), "A numerical-integration perspective on Gaussian filters", IEEE Transactions on Signal Processing, 54(8), 2910-2921.

[17] I. Arasaratnam, S. Haykin, amd T. Hurd, (2010). Cubature Kalman Filtering for Continuous-Discrete Systems: Theory and Simulations. Signal Processing IEEE, 58(10), 4977-4993.

[18] S. Julier, and J. Uhlmann, "A General Method for Approximating Nonlinear Transformations of Probability Distributions", Dept. of Engineering Science, University of Oxford, Tech. Rep., 1996.

[19] T.D. Gillespie, "Fundamentals of vehicle dynamics," SAE, ISBN 156091-199-9, 1992. 